EDITORIAL

\title{
Cardiac effects of anabolic steroids
}

\author{
J R Payne, P J Kotwinski, H E Montgomery
}

Heart 2004;90:473-475. doi: 10.1136/hrt.2003.025783

Anabolic steroid abuse in athletes has been associated with a wide range of adverse conditions, including hypogonadism, testicular atrophy, impaired spermatogenesis, gynaecomastia, and psychiatric disturbance. But what effect does steroid abuse have on the cardiovascular system?

See end of article for authors' affiliations

.....................

Correspondence to:

Dr H E Montgomery,

British Heart Foundation

Laboratories, Royal Free \&

University College Medical

School, Rayne Building, 5

University Street, London

WCIE 6JF, UK;

h.montgomery@ucl.ac.uk

.................... eft ventricular hypertrophy (LVH) indepen-morbidity across diverse disease states. ${ }^{1}$ While cardiac diastolic or contractile failure might result directly from structural change within the ventricle (such as altered capillary density or matrix deposition), the association of LVH with cardiovascular disease is more likely dependent upon the increased activity of shared physiological pathways driving both processes. The nature of these underlying mechanisms remains poorly understood. In this regard, escalating attention has focused on the potential role of steroid hormones on LV growth responses.

Whether of local or systemic origin, endogenous steroid hormones appear to drive LV growth. Systemic glucocorticoid excess is associated with significant hypertrophy. This action is more likely to be direct, rather than mediated through an elevated pressor burden, ${ }^{2}$ with aldosterone having similar effects. ${ }^{3}$ Local myocardial reninangiotensin systems (RAS) play a role in regulating LV growth, ${ }^{4}$ and at least part of the hypertrophic responses to steroid hormones may be mediated through upregulation of local RAS expression. ${ }^{5}$ Anabolic/androgenic steroids (AAS-primarily comprising testosterone and its synthetic derivatives) are likely to share such influences on the LV hypertrophic response through actions on the androgen receptor (AR), a transcriptional regulator. ${ }^{67}$ Indeed, ARs are almost ubiquitously expressed, being found not only in skeletal muscle cells, but also on cardiac myocytes. Several lines of evidence also implicate endogenous androgenic pathways in the development of cardiac hypertrophy, including the demonstration of raised $5 \alpha$ reductase, aromatase, and AR expression in hypertrophic hearts of both humans and mice. ${ }^{\circ}$

\section{EXOGENOUSLY ADMINISTERED STEROIDS}

Given these putative effects of steroid hormones (and AAS in particular) on LV growth, we might dently predicts cardiovascular mortality and expect exposure to exogenously administered steroid hormones to be associated with an exaggerated LV hypertrophic response to any other hypertrophic stimulus.

Exercise is just such a potent cardiac hypertrophic stimulus. ${ }^{49}$ Meanwhile, athletes are increasingly exposing themselves to supra-physiological doses of AAS. These are known to increase skeletal muscle mass and strength ${ }^{7}-$ effects which form the basis for their administration to enhance athletic performance. A variety of AAS are often taken simultaneously (so called "stacking"), and in doses which result in 10-100 fold increases in androgen concentrations. ${ }^{7}$ Administration regimens usually involve a 6-12 week cycle and are often administered in a "pyramidal" fashion, with doses tapering from low to high to low..$^{10}$ Abused substances include testosterone, its $17-\beta$ esters, and those based on modified steroid rings (including $17-\alpha$ derivatives). ${ }^{7}$

The largest group to make such use of AAS are the very group whose LVH response to exercise is likely to be the greatest - the strength or resistance training (RT) athletes. One study from 1995 suggested that two thirds of elite US powerlifters have self reported use of AAS to enhance performance ${ }^{11}$; even "dope testing" may be underestimating the true extent of such use. ${ }^{12}$ What evidence is there that AAS administration enhances the LV hypertrophic response to resistance exercise?

In this issue of Heart, Urhausen and colleagues report the results of a cross sectional study of cardiac morphology in relation to AAS use. ${ }^{13}$ Male bodybuilders/powerlifters currently using AAS or ex-users who had abstained from AAS exposure for over 12 months ( $U$ and ExU, $\mathrm{n}=17$ and 15 , respectively) were compared to 15 weightlifters who denied current or past use of AAS (WL). Left ventricular wall thickness and cavity dimensions were assessed using echocardiography, and muscle mass (LVMM) calculated using the Devereux equation. Absolute LVMM measures (mean (SD)) were significantly greater for U than ExU or WL (281 (54) g v 232 (42) g v 204 (44) g for U $v$ ExU $v$ WL, respectively), with differences between ExU and WL only reaching significance after adjustment for body surface area or fat-free mass. These results suggest that AAS use increases the LV hypertrophic response

Abbreviations: AAS, anabolic/androgenic steroids; AR, androgen receptor; ExU, ex-users of anabolic steroids; hCG, human chorionic gonadotrophin; $h G H$, human growth hormone; LVH, left ventricular hypertrophy; LVMM, left ventricular muscle mass; RAS, reninangiotensin system; $R T$, resistance training; $U$, users of anabolic steroids; WL, weightlifters 
to exercise, an effect which might last for well over a year.

\section{CAUTION NEEDED}

Such data must nonetheless be treated with caution. We know, for example, that the magnitude and pattern of hypertrophy is dependent on the nature, duration, and intensity of exercise undertaken. ${ }^{8}{ }^{14}$ Thus, strength trained athletes (such as weightlifters, powerlifters, bodybuilders, and throwers) develop a greater increase in wall thickness, a more concentric pattern of LV growth, and a lesser increase in LV chamber internal dimensions ${ }^{8}$ in comparison to those undergoing predominantly aerobic/endurance exercise. In the study under discussion, training patterns will have varied. One might suspect that subjects taking AAS were also the most motivated to train (whether by initial predisposition, or psychological impact of the steroid use itself). However, this does not seem to be the case as the authors report that the magnitude of training did not differ between U, ExU, and WL groups. Even so, more subtle differences in training pattern may have existed between bodybuilders, powerlifters, and weightlifters. Although all groups lift exceptionally heavy weights, the total load and training pattern are likely to differ.

Other factors may also have been of influence. Diet (including the use of supplements) may have differed between groups, as might the use of other agents. Abusers of AAS frequently also self administer other drugs including stimulants, antioestrogens, human chorionic gonadotrophin (hCG), and human growth hormone (hGH). ${ }^{10}$ It is unclear to what extent these and other drugs might have driven LV growth, and whether the ExU group were still taking any of these. Neither can mechanistic inferences be drawn from the data: the putative effects of AAS on LV growth may have been mediated directly, or through secondary phenotypes such as alterations in circulating volume or blood pressure. Certainly, resting systolic blood pressure is higher in the $\mathrm{U} v$ ExU group, a difference which persists as a trend for exercising blood pressure. The use of such drugs (as well as differences in patterns of training) may also have influenced fat-free mass and body surface area. The adjustment for such anthropometric measures may have contributed to the significance of the comparison between ExU and WL.

Finally, it is noticeable that the ExU group were younger than the $\mathrm{U}$ group, and it may be that LV growth responses differ with subject age. Nonetheless, these data are consistent with existing data. Over a decade ago, De Piccoli demonstrated that LV mass among bodybuilders who used AAS was greater than that in non-users, and did not regress over a nine week period of abstinence. ${ }^{15}$

\section{HEALTH IMPLICATIONS}

If AAS use is associated with an exaggerated LV hypertrophic response to training, what are the likely health implications? They may be profound. In terms of non-cardiac morbidity, AAS use is associated with hypogonadism, testicular atrophy, impaired spermatogenesis, baldness, acne, gynaecomastia, and psychiatric disturbance. Such drugs also have toxic effects on metabolic profile and hepatic structure and function, ${ }^{10}$ as well as potentially promoting neoplastic growth. ${ }^{10}$ Indeed, Parsinnen reported the 12 year mortality to be $12.9 \%$ among 62 male powerlifters suspected of AAS use, compared to $3.1 \%$ in a control population. ${ }^{16}$

LVH is an independent risk factor for cardiovascular mortality and (through whatever mechanism) one might anticipate an excess cardiovascular mortality among AAS users in whom LVH occurs. In addition, the recognised association of AAS use with hypertension and dislipidaemia (raised low density lipoprotein and reduced high density lipoprotein cholesterol, and raised triglycerides), ${ }^{10}$ as well as influences on coagulation and platelet aggregation, ${ }^{10}$ might increase such risk. While it is debatable whether ASS use is indeed associated with an increased risk of premature cardiovascular death, $38 \%$ of the deaths in Parssinen's powerlifting group were attributed to "myocardial infarction", ${ }^{16}$ while several case reports have attributed myocardial infarction in athletes to ASS abuse.

In some cases, infarction has occurred without evident coronary thrombosis or atherosclerosis, leading to the hypothesis that ASS may induce coronary vasospasm in susceptible individuals. ${ }^{10}$ Similarly there are several case reports of increased thromboembolic risk. ${ }^{10}$ In a recent postmortem series of 34 AAS abusers aged 20-45 years (comprising 12 homicides, 11 suicides, 12 "accidental" deaths, and two of indeterminate cause), 12 of the deceased showed cardiac pathology. Findings included hypertrophy (7 cases), myocardial or endocardial fibrosis (5), cardiac steatosis (1), myocardial coagulation necrosis (2), and coronary atheroma (4). Cardiac changes were adjudged to have contributed to death by poisoning in two cases. ${ }^{17}$ However mediated, such a morbid burden is likely to rise with time. The US National Institute on Drug Abuse reported in 1999 that between $2.7-2.9 \%$ of year 8-12 high school teenagers had experimented at least once with AAS, representing a $38-50 \%$ rise since $1991 .^{18}$

The influence of steroid hormones on the heart thus warrants further study. Evidently, the potential impact of steroid abuse on public health is a matter of concern. Perhaps more importantly, however, such studies might lead to a greater understanding of the shared mechanisms through which cardiac growth and cardiovascular disease are mediated. Such issues are increasingly exciting as the identification of local myocardial steroid synthesis (and its potential pathogenicity ${ }^{19}$ ) is paralleled by the demonstrated efficacy of steroid antagonists in cardiac disease.$^{20}$ We might yet see a role for steroid antagonists such as aldosterone in the primary or secondary prevention of LVH, and its associated cardiovascular sequelae.

\section{ACKNOWLEDGEMENTS}

JRP is funded by the British Heart Foundation (PG/02/021), who also provide core funding for the Centre for Cardiovascular Genetics. PJK and HEM are funded by the Portex Endowment. HEM is a Portex Senior Lecturer in Cardiovascular Genetics.

\section{Authors' affiliations}

J R Payne, P J Kotwinski, H E Montgomery, Centre for Cardiovascular Genetics, British Heart Foundation Laboratories, London, UK

\section{REFERENCES}

1 Levy D, Garrison R, Savage D, et al. Prognostic implications of echocardiographically determined left ventricular mass in the Framingham heart study. N Engl J Med 1990;322:1561-6.

2 Fallo $\mathrm{F}$, Budano S, Sonino N, et al. Left ventricular structural characteristics in Cushing's syndrome. J Hum Hypertens 1994;8:509-13.

3 Delles C, Schmidt BM, Muller HJ, et al. Functional relevance of aldosterone for the determination of left ventricular mass. Am J Cardiol 2003;91:297-301.

4 Montgomery HE, Clarkson P, Dollery CM, et al. Association of angiotensinconverting enzyme gene I/D polymorphism with change in left ventricular mass in response to physical training. Circulation 1997;96:741-7.

5 Dostal DE, Booz GW, Baker KM. Regulation of angiotensinogen gene expression and protein in neonatal rat cardiac fibroblasts by glucocorticoid and beta-adrenergic stimulation. Basic Res Cardiol 2000;95:485-90.

6 Liu PY, Death AK, Handelsman DJ. Androgens and cardiovascular disease. Endocr Rev 2003;24:313-40.

7 Kuhn CM. Anabolic steroids. Recent Prog Horm Res 2002;57:411-34.

8 Pluim BM, Zwinderman AH, van der Laarse A, et al. The athlete's heart. A meta-analysis of cardiac structure and function. Circulation 2000; 101:336-44.

9 Haykowsky MJ, Dressendorfer R, Taylor D, et al. Resistance training and cardiac hypertrophy: unravelling the training effect. Sports Med 2002;32:837-49.

10 Parssinen $M$, Seppala T. Steroid use and long-term health risks in former athletes. Sports Med 2002;32:83-94. 
11 Wagman DF, Curry LA, Cook DL. An investigation into the annabolic androgenic steroid use by elite U.S. powerlifters. J Strength Cond Res 1995;9:149-54.

12 Curry LA, Wagman DF. Qualitative description of the prevalence and use of anabolic androgenic steroids by United States powerlifters. Percept Mot Skills 1999:88:224-33.

13 Urhausen A, Albers T, Kindermann W. Are the cardiac effects of anabolic steroid abuse in strength athletes reversible? Heart 2004;90:496-501.

14 Urhausen A, Kindermann W. Sports-specific adaptations and differentiation of the athlete's heart. Sports Med 1999;28:237-44.

15 De Piccoli B, Giada F, Benettin A, et al. Anabolic steroid use in body builders: an echocardiographic study of left ventricle morphology and function. Int J Sports Med 1991;12:408-12.
16 Parssinen $M$, Kujala U, Vartiainen E, et al. Increased premature mortality of competitive powerlifters suspected to have used anabolic agents. Int I Sports Med 2000;21:225-7.

17 Thiblin I, Lindquist O, Rajs J. Cause and manner of death among users of anabolic androgenic steroids. J Forensic Sci 2000:45:16-23.

18 NIDA. NIDA Community Drug Alert Bulletin - Anabolic Steroids. National Institute on Drug Abuse; $h+t p: / / 165.112 .78 .61 /$ SteroidAlert/ SteroidAlert.html (accessed 22 September 2003).

19 Rocha R, Funder JW. The pathophysiology of aldosterone in the cardiovascular system. Ann N Y Acad Sci 2002;970:89-100.

20 RALES Investigators. Effectiveness of spironolactone added to an angiotensinconverting enzyme inhibitor and a loop diuretic for severe chronic congestive heart failure (the randomized aldactone evaluation study [RALES]). Am J Cardiol 1996:78:902-7.

\section{IMAGES IN CARDIOLOGY}

\section{EX filter wire usage in stenting right coronary artery lesion with diffuse aneurysmal dilatation}

A 63 year old man was transferred to our hospital in an emergency following the onset of chest pain two hours previously. The ECG showed normal sinus rhythm with ST segment elevation in the anterolateral leads. He had a history of active Crohn's disease.

An urgent cardiac catheterisation revealed a 99\% mid-left anterior descending artery (LAD) stenosis (culprit lesion) and a 99\% stenosis of an aneurysmal right coronary artery (RCA) (panel A). It was decided to attempt percutaneous coronary intervention (PCI). A glycoprotein IIb/IIIa inhibitor was not administered because of the bleeding risk related to Crohn's disease. The LAD lesion was predilated and a stent $2.75 \mathrm{~mm} \times 33 \mathrm{~mm}$ (Cypher, Cordis) was implanted with a TIMI grade 3 flow. Subsequently it was decided to treat the RCA stenosis. In order to avoid distal embolisation from mural thrombus within the ectatic segment, we opted to use a distal protection device (Filter Wire EX, Boston Scientific). Until now distal protection systems have been mainly used in the setting of acute coronary syndromes, and to treat thrombotic or degenerated saphenous vein graft stenosis.

After positioning the distal protection device, the RCA lesion was predilated with a $4.0 \times 20 \mathrm{~mm}$ Maverik balloon at $12 \mathrm{~atm}$. A $5.0 \times 18 \mathrm{~mm}$ Express stent was then deployed at 16 atm. A massive embolisation occurred during angioplasty and stent implantation, resulting in a partial occlusion of the filter system but without distal embolisation (panel B). When the filter was retrieved a TIMI grade 3 distal flow was achieved and the no-reflow phenomenon did not occur (panel C). Inspection of the filter revealed the presence of many particles (panel D) which, at histological examination, appeared to be composed of fibrin, cholesterol crystal, foam cells, and amorphous material. These findings suggest that a large piece of intimal debris peeled off and was dislodged from the main plaque during the interventional procedure. The patient's subsequent clinical course was uncomplicated.

The use of distal protection devices in the treatment of an RCA lesion within a diffuse ectatic segment, even in the angiographic absence of apparent thrombus, is suggested.

M Fineschi, A ladanza, C Pierli, A Bravi orfine@tin.it
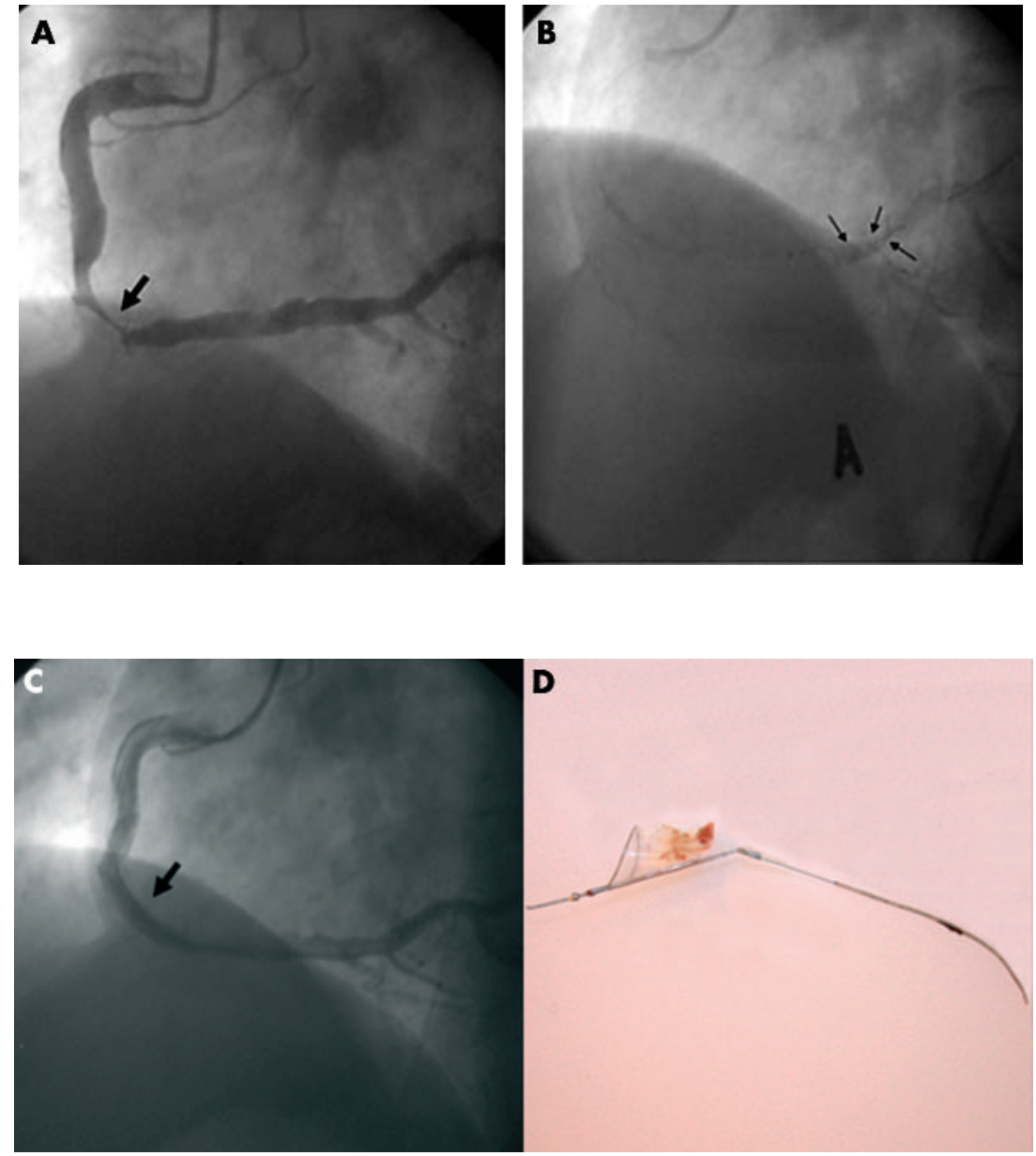